\title{
Arbor
}

\section{El Área de Ciencia y Tecnologías Químicas del CSIC: ¿un Área de químicas?}

\author{
María Teresa García-López, Pilar Goya Laza \\ Con la colaboración de Pilar Erra Sarabasa, María José \\ González Carlos, María Luisa Jimeno Herranz, María \\ Desamparados Mifsud Corts, Ofelia Nieto López, \\ Carmen Ochoa de Ocáriz Herrero, Soledad Penadés \\ Ullate, Inmaculada Rodríguez Ramos
}

Arbor CLXXII, 679-680 (Julio-Agosto 2002), 501-517 pp.

El artículo titulado "El Area de Ciencia y Tecnologías Químicas del CSIC: ¿̇un Area de quimicas?» pretende mostrar una breve panorámica del lugar que ocupa el género femenino dentro de la citada Area. En la primera parte se aportan las estadísticas que reflejan el número y porcentaje de científicas totales en los distintos Institutos del Area, así como su distribución entre las tres escalas de personal científico del Organismo. Una segunda parte está dedicada a recoger las aportaciones personales de varias científicas del Area acerca del tema, visto desde sus propias experiencias.

\section{Introducción}

Para tratar de dar una visión, lo más cercana posible a la realidad, referente a la situación actual de las mujeres en la plantilla del personal científico del Area de Ciencia y Tecnologías Químicas, hemos considera- 
do apropiado comenzar este artículo presentando, en primer lugar, una tabla que recoge cuantitativamente el número de científicas totales en los distintos Institutos del Area, así como el reparto de éstas en las tres escalas de personal científico del Organismo (Tabla 1). Cuatro diagramas visualizan los datos recogidos en la tabla (Figuras1, 2, 3 y 4). Seguidamente, hemos intentado traducir a palabras el lenguaje numérico de los datos estadísticos (Apartados 2 y 3) para finalizar con el apartado que recoge diversas opiniones personales aportadas por distintas científicas del Area.

\section{2. ¿Hablan las estadísticas?}

Con el fin de dar obviamente una respuesta afirmativa a esta pregunta, hemos tratado de eliminar aquellos datos que pudieran distorsionar las deducciones extraibles a partir de ellos. Este es el caso del Instituto de Ciencia de Materiales de Aragón (ICMA, Zaragoza) y del Laboratorio de Investigación en Tecnologías de la Combustión (LITEC, Zaragoza), cuyo personal científico total dentro del Area es muy escaso. $\mathrm{Al}$ mismo tiempo, el bajo valor de algunas de las muestras aconseja no sacar deducciones puntuales de la comparación entre los diez Institutos reflejados en estas estadísticas. Así, no podríamos afirmar que el Instituto de Tecnología Química ( ITQ, Valencia) y el Instituto de Investigaciones Químicas (IIQ, Sevilla) son aquellos en los que exista una discriminación hacia la mujer superior a la media, ya que, precisamente, son aquellos que cuentan con un número menor de científicos totales. Es más, se caería en una clara contradicción al observar el absoluto equilibrio entre géneros en la escala de investigadores del IIQ. Tampoco parece razonable sacar conclusiones acerca de los Institutos en los que las mujeres parecen haberse promocionado más fácilmente, ya que el número de investigadores y profesores es, en general, tan escaso, independientemente del sexo, que una diferencia de uno, o de una, dispara los porcentajes. Quizá el único dato a reseñar es la elevada presencia de mujeres en el Instituto de Química Médica (IQM, Madrid ), que supera a la de hombres en las tres escalas. Recogiendo los comentarios de la Directora actual de este Instituto acerca de esta particularidad, única entre todos los Institutos del Area «en sus orígenes, año 1974, y siguiendo la tónica general del CSIC, existía en el IQM una mayor proporción de científicos que científicas. En el año 1991 hay un punto de inflexión y, desde entonces, el número de éstas supera al de científicos masculinos. No podemos analizar en profundidad las causas del hecho de que, en la 


\section{El Área de Ciencia y Tecnología Químicas del CSIC}

actualidad, seamos un Instituto con predominio del género femenino en todas las escalas de investigación. Probablemente las causas son múltiples, entre otras se podrían destacar el hecho de ser un Instituto joven y el que su fundador y primer Director, Profesor Ramón Madroñero, tuviera un talante aperturista y nunca discriminara a nadie por razón de su género. Otras causas serían las bajas consecutivas de investigadores hombres por razones de muerte, jubilación, traslado a empresas farmacéuticas o a otros organismos de la administración, unido a que, durante los últimos años, han sido únicamente mujeres las que han accedido a los puestos en plantilla. Sin embargo, quiero destacar que el Instituto de Química Médica cuenta con una altísima producción científica, demostrándose así el hecho de que el Instituto esté formado por un porcentaje elevado de mujeres, no es óbice, muy al contrario, para que su rendimiento científico, cuantitativo y cualitativo, sea muy satisfactorio. Una prueba clara de ello es que uno de los grupos participantes en el proyecto que ha obtenido el Premio Descartes 2001 de la UE para la excelencia científica y tecnológica mediante la investigación en colaboración europea, es un grupo de este Instituto compuesto en su totalidad por mujeres».

A la vista de estos datos y de estos comentarios referentes al IQM cabría, como mínimo, reflexionar, y hasta dudar, del estereotipo sobre la menor competitividad de las mujeres respecto a la de los hombres, sugerida por algunas investigadoras del Area para explicar el menor número de féminas en las dos escalas superiores. Con la salvedad del pequeño valor de la muestra, el número de investigadores mujeres en el Instituto de Carboquímica (ICB, Zaragoza ) es también favorable al género femenino $(66,7 \%)$.

Figura 1. Científicos totales (\%)

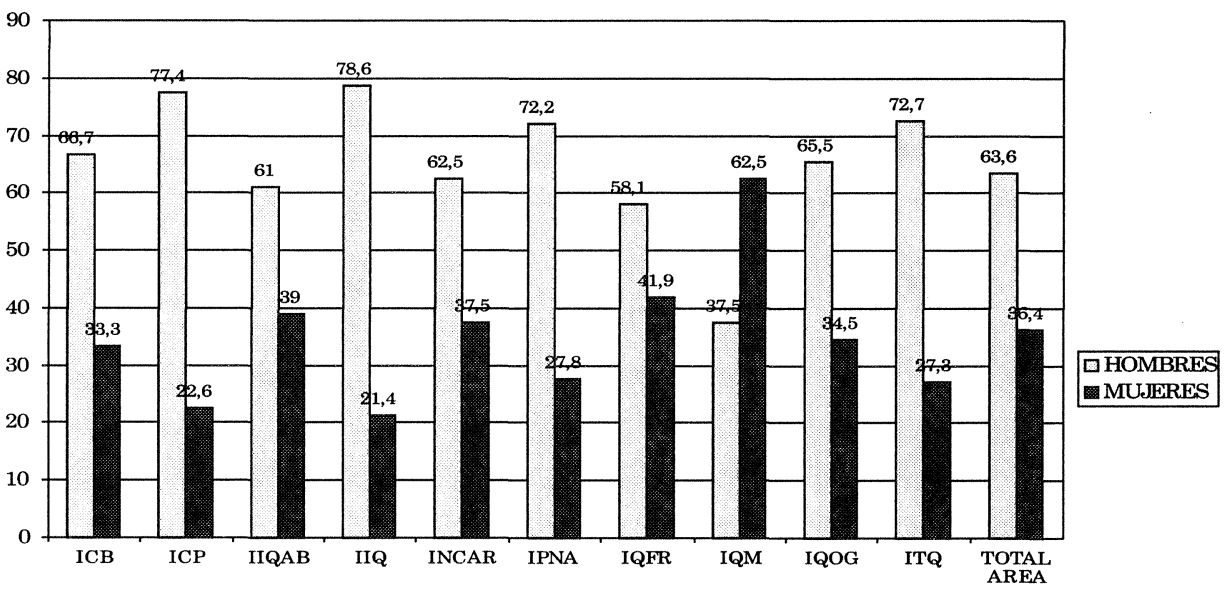


TABLA 1. Area de Ciencia y Tecnologias Químicas ${ }^{\mathrm{a}}$

\begin{tabular}{|c|c|c|c|c|c|c|c|c|}
\hline Institutos $^{\mathbf{b}}$ & \multicolumn{2}{|c|}{ Cientifico titular } & \multicolumn{2}{|c|}{ Investigador Cientifico } & \multicolumn{2}{|c|}{ Profesor de Investigación } & \multicolumn{2}{|c|}{ Científicos totales } \\
\hline & H. + M. ${ }^{c}$ & M. (\%) & H. + M. ${ }^{\mathrm{c}}$ & M. (\%) & H. + M. ${ }^{\mathbf{c}}$ & M. (\%) & H. + M. ${ }^{c}$ & M. (\%) \\
\hline ICB & 15 & $4(22,7)$ & 3 & $2(66,7)$ & - & - & 18 & $6(33,3)$ \\
\hline ICP & 17 & $5(29,5)$ & 6 & $2(33,3)$ & 8 & $-(0)$ & 31 & $7(22,6)$ \\
\hline IIQAB & 26 & $13(50,0)$ & 6 & $2(33,3)$ & 9 & $1(11,1)$ & 41 & $16(39,0)$ \\
\hline IIQ & $10^{d}$ & $2(20,0)$ & 2 & $1(50,0)$ & 2 & $-(0)$ & $14^{\mathrm{d}}$ & $3(21,4)$ \\
\hline INCAR & 19 & $8(42,1)$ & 3 & $1(33,3)$ & 2 & $-(0)$ & 24 & $9(37,5)$ \\
\hline IPNA & 11 & $5(45,4)$ & 5 & $-(0)$ & 2 & $-(0)$ & 18 & $5(27,8)$ \\
\hline IQFR & 21 & $13(61,9)$ & 15 & $4(26,7)$ & 7 & $1(14,3)$ & 43 & $18(41,9)$ \\
\hline IQM & 14 & $9(64,3)$ & 7 & $4(57,1)$ & 3 & $2(66,7)$ & 24 & $15(62,5)$ \\
\hline IQOG & 15 & $7(46,7)$ & 9 & $3(33,3)$ & 5 & $-(0)$ & 29 & $10(34,5)$ \\
\hline ITQ & 6 & $2(33,3)$ & 3 & $1(33,3)$ & 2 & $-(0)$ & 11 & $3(27,3)$ \\
\hline Total & 154 & $68(44,1)$ & 59 & $20(33,9)$ & 40 & $4(10,0)$ & 253 & $92(36,4)$ \\
\hline
\end{tabular}

- Datos a 31 de diciembre de 2001.

ICB: Instituto de Carboquímica.-ICP: Instituto de Catálisis y Petroleoquímica.-IIQAB: Instituto de Investigaciones Químicas y Ambientales.-IIQ: Instituto de Investigaciones Químicas.-INCAR: Instituto Nacional del Carbón.-IPNA: Instituto de Productos Naturales y Agrobiología.-IQFR: Instituto de Química Física Rocasolano.-IQM: Instituto de (ICMA) ni el Laboratorio de Investigación en Tecnologías de la Combustión (LITEC), ya que el escaso personal Científico total en sus plantillas, 3 y 4 Científicos respectivamente, podría

- desvirtuar, a efectos de esta estadística, los resultados.
H,: Hombres. - M.: Mujeres.

Se han incluido a tres Profesores Titulares de Universidad, pertenecientes a este Instituto, dentro del cómputo de Científicos Titulares. 


\section{El Área de Ciencia y Tecnología Químicas del CSIC}

Figura 2. Científicos titulares

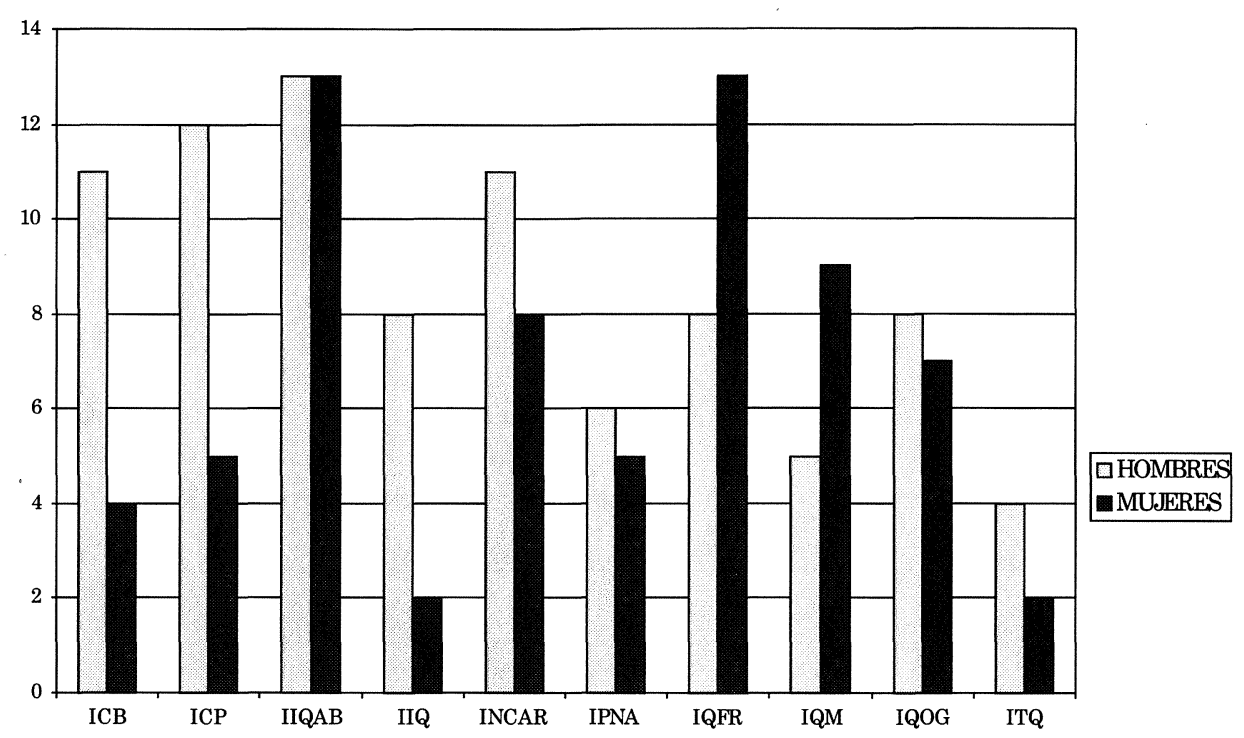

FiguRA 3. Investigadores Científicos

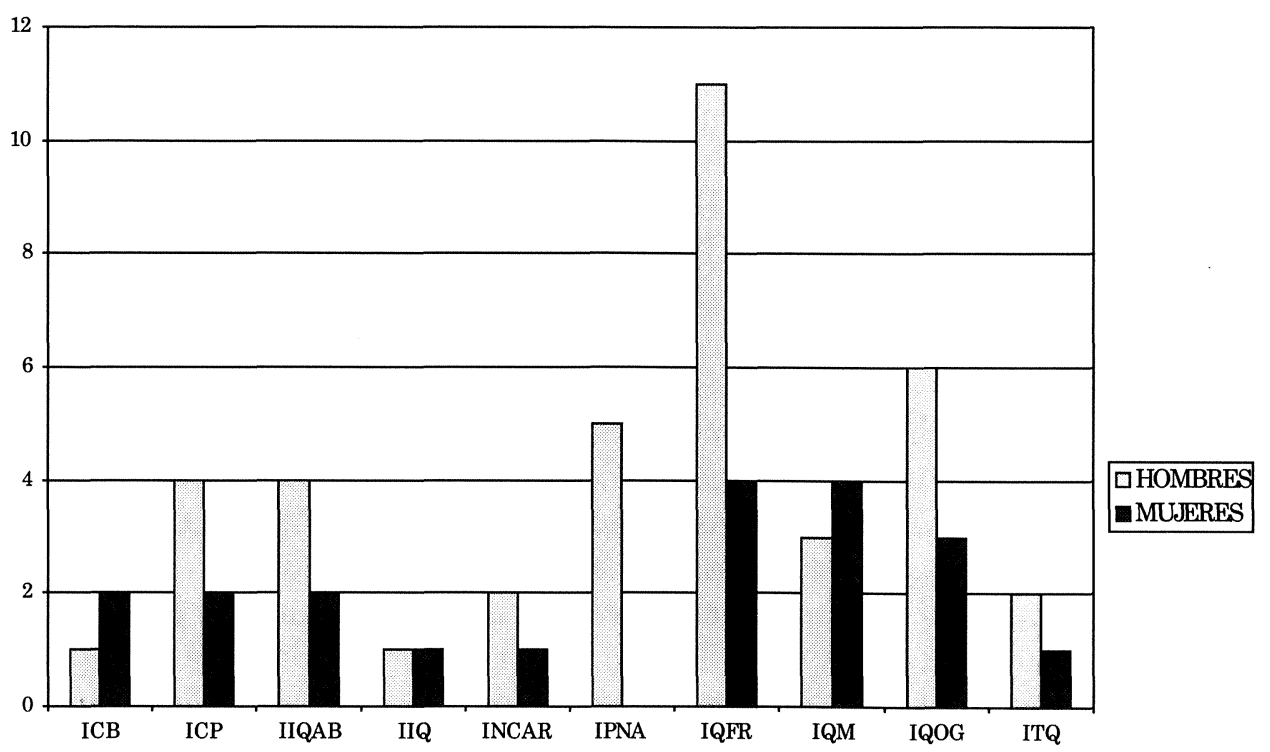


FiguRA 4. Profesores de Investigación

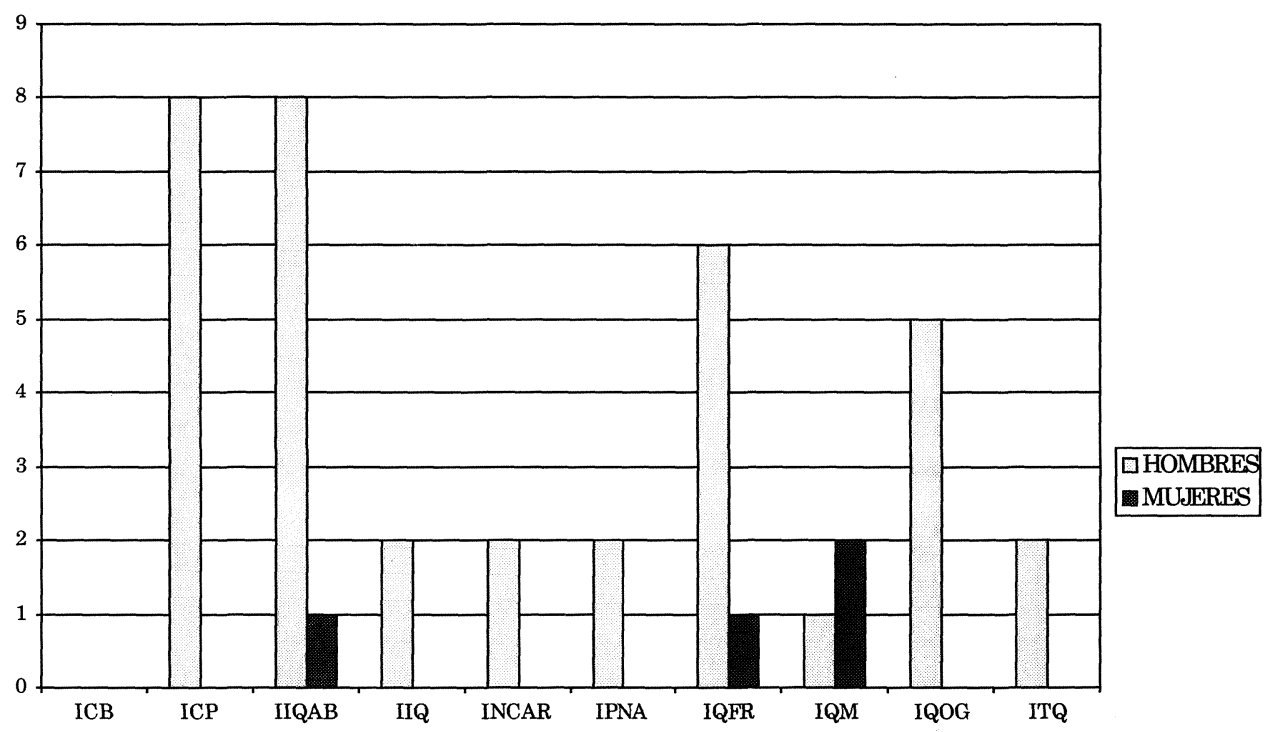

\section{3. ¿Qué dicen las estadísticas?}

El porcentaje total de científicas en el Area de Química (36,4 por 100) pone de manifiesto un desequilibrio desfavorable para las mujeres. Sin embargo, no se pueden considerar ningún tipo de argumentos que expliquen este desequilibrio específicamente para este Area, ya que es muy similar al existente en la mayoría de las Areas del CSIC restantes y, en todo caso, se sitúa en las Areas menos desfavorecidas para la mujer. Tampoco parece deducirse diferencias concluyentes ni entre las dos vertientes del Area - Ciencia y Tecnología- ni entre las distintas orientaciones hacia otras Areas -Física o Biología- de los distintos Institutos.

El hecho que sí evidencian los datos aportados es el descenso significativo en el desequilibrio, según se desciende en la categoría de las tres escalas, que se podría calificar de espectacular si se compara el $10 \%$ total de Profesoras de Investigación en el Area con el 44,1 \% de Científicas Titulares. Como reflejan varias de las aportaciones personales recogidas, el progresivo cambio en el contexto social acaecido en nuestro país en los últimos treinta años es, indudablemente, un factor de muy elevado peso. Tampoco deberíamos olvidar que el cambio de mentalidad en las parejas hacia una igualdad entre hombres y mujeres ha venido unido, o, ha es- 


\section{El Área de Ciencia y Tecnología Químicas del CSIC}

tado favorecido, por un cambio en las necesidades económicas familiares (la aportación de dinero se va independizando del género).

En resumen, estos resultados estadísticos con sus inevitables defectos, apuntan hacia que ni la Ciencia, en general, ni la Ciencia y Tecnologías Químicas, en particular, con sus diferentes ramas no muestran características diferenciales en función del sexo. Aunque aún hoy persiste una menor presencia femenina en el ámbito investigador del Area, si asumimos que el menor desequilibrio que se observa en la escala «más jóven»-Científicos Titulares (44,1\%) — es consecuencia del mencionado cambio social, dentro de algún tiempo no tendrán lugar artículos o debates para razonar o demostrar el género de la Ciencias y Tecnologías Químicas. Como el sexo de los Angeles.

\section{Aportaciones personales}

A continuación vamos a intentar resumir las aportaciones personales que sobre el tema nos han enviado distintas científicas del Area de Química. Sin ánimo de ser una muestra estadísticamente significativa, la selección fue realizada de forma que incluyera científicas pertenecientes a Institutos del Area de distinta naturaleza (básicas y tecnológicas), de diferente tamaño, situados en distintas Comunidades Autónomas, y de las tres escalas científicas, si bien, en lo que se recoge a continuación hay un predominio de opiniones de mujeres que llevan en el CSIC un cierto número de años.

En líneas generales hay acuerdo en que la discriminación entre mujeres y hombres ha disminuido respecto a épocas anteriores. Haciendo un poco de historia, según nos recuerda una colega del Area, «en tiempos pasados era evidente la existencia de un cierto antagonismo entre mujer y ciencia. Un concepto muy subjetivo que colocaba a la mujer en un marco de protagonismo dentro del área de Servicios (Administración, Secretariado, Ayudantías Técnicas, etc.), pero no se le concedía fiabilidad en el campo de las actividades creativas, relegando en cierto modo su potencialidad a labores que exigían una menor capacidad de concentración. Un círculo vicioso del que no sería justo culpar únicamente a los hombres, como progenitores de una sociedad machista.

El concepto que prevalecía en la sociedad de nuestros abuelos respecto de la mujer, como modelo exclusivo de esposa y madre, parecía incompatible con el de mujer profesional, y mucho más con el de mujer científica. Las pocas mujeres que lograron situarse al mismo nivel que los hombres, en el orden jerarquizado de la intelectualidad, tenían a veces 
reflejada en su apariencia la dureza de los esfuerzos realizados para alcanzar esa meta. En general, se les calificaba de "poco femeninas"».

Respecto a la evolución de la situación, una investigadora hace el siguiente análisis: «La mujer científica en el trabajo profesional, tanto en la industria como en instituciones oficiales, es actualmente mucho más respetada y considerada que años atrás. La mujer con su actitud de responsabilidad frente al trabajo ha logrado poco a poco que las diferencias de consideración (como poseer capacidad suficiente, formación profesional, dotes de mandos, efectividad, salario) entre hombres y mujeres se haya reducido considerablemente. La mujer ha tenido que demostrar constantemente su valía, y ello, en cierto modo, ha contribuido a un cambio de mentalidad en la sociedad.

En los años sesenta y setenta, prosigue esta investigadora, « a igualdad de cualidades, el puesto de un trabajo se asignaba automáticamente a un hombre, dada la mentalidad de los hombres y mujeres de aquella época. Se consideraba que la misión de la mujer era ser esposa, madre y ama de casa. El hombre tenía que aportar el pan a la familia, y por tanto la sociedad le tenía que favorecer. Uno de los inconvenientes más destacable para que una mujer fuera aceptada para ocupar un puesto de trabajo en la industria era, y aún es, aunque menos frecuentemente, la posible maternidad, o sea, la posible ausencia en el trabajo. Así, una de las preguntas que formulaba el seleccionador del trabajo era: ¿Tienes novio? Ello era menos acusado en las Instituciones oficiales, pero las plazas, a igualdad de conocimientos científicos, eran más fácilmente ocupadas por los hombres que por las mujeres. Además, no se soportaba que una mujer pudiera ocupar un puesto de trabajo de mando superior al del hombre». Y concluye : "Situándonos en los años ochenta y noventa las discriminaciones antes mencionadas entre hombre y mujer han ido disminuyendo debido posiblemente al cambio de mentalidad generacional y el haber demostrado la mujer su capacidad para realizar con éxito su trabajo. También cabe considerar que el número de mujeres con la formación y capacidad adecuadas para ejercer investigación científica ha aumentado considerablemente».

¿Cuál es la situación actual? En este punto hay diversidad de opiniones. Hay quien piensa que «actualmente la discriminación entre hombre y mujer prácticamente no tiene lugar. La mentalidad ha cambiado lo suficientemente como para que se valore, en primicia, la capacidad científica de las personas frente al sexo», y quien opina que «el hecho de que el número de mujeres científicas, políticas, empresarias, etc., sea inferior al de hombres se debe más bien a que existen otras opciones, que quizás sean más cómodas o más atractivas para un gran número de mujeres, o 


\section{El Área de Ciencia y Tecnología Químicas del CSIC}

quizás también a una carencia de objetivos profesionales concretos planteados a su debido tiempo; es decir, en las etapas de formación previas a la toma de decisiones (estudios primarios, bachiller, incluso carrera universitaria)», y que «en el tiempo presente no existe campo de actividad que esté vedado para la mujer. Lo que ocurre es que para llegar a poder asumir grandes responsabilidades el camino es duro, y no todas las mujeres tienen la preparación suficiente o no están dispuestas a seguirlo. Muchas consideran todavía que el papel más importante que pueden jugar en la vida es el de esposa y madre, y ésta es una alternativa, innegablemente, muy respetable», y quien concretando respecto al Area de Ciencia y Tecnología Química comenta lo siguiente: «Después de veinte años trabajando en investigación, primero como doctoranda y postdoctoranda, y posteriormente como responsable de un grupo de investigación relativamente pequeño (lo integramos una media de 10 personas) he llegado a una serie de conclusiones que a continuación te enumero.

Creo, sin pecar de optimismo, que el CSIC en general, y en particular el Area de Ciencia y Tecnologías Químicas, donde he desarrollado mi carrera, no establece, en principio, a la hora del acceso, diferencias entre hombres y mujeres. Ahora bien, la carrera científica es muy competitiva y requiere un gran esfuerzo y dedicación el mantenerse y poder progresar en ella. Esto último hace que cuando las mujeres tienen una carga familiar (hijos) sean sus condiciones particulares (reparto de tareas con la pareja, ayuda de otros familiares, etc.) las que condicionen la evolución de su carrera científica».

Hay otras científicas que sí sienten que existe una cierta discriminación . Según una de ellas: «Personalmente tengo que distinguir dos niveles con respecto a mi integración en el mundo de la Ciencia y Tecnología (CyT) en general, y el CSIC en particular. Primero, el nivel institucional, donde no creo haber tenido ningún problema para integrarme en el mundo profesional. Pude estudiar, hacer mi Tesis Doctoral, sacar unas oposiciones al CSIC, etc., sin haber percibido ninguna discriminación. En todo caso, si la hubiera percibido no habría influido en mis decisiones. Primero, porque mis retos y opciones personales me los impongo yo y nadie más. Segundo, porque la legislación española me ampara no haciendo distinciones entre sexos. Muy distinto es en el nivel del día a día. Para resumir esta idea puedo decir que como profesional no existo para prácticamente la totalidad de mis compañeros de profesión, sean del CSIC o de la Universidad, y creo que esta sensación puede hacerse extensiva a todas las mujeres que estamos incluidas en el sistema de CyT. Los compañeros químicos españoles nunca me han invitado a exponer mi trabajo en ningún foro científico, nunca han pedido mi opinión sobre proble- 


\section{María Teresa García-López, Pilar Goya Laza}

mas referentes a Ciencia e Investigación, nunca se han interesado por mi trabajo, y si alguna vez lo han hecho ha sido a través de la "recomendación especial de un colega del género masculino de prestigio reconocido». Podría enumerar una serie de anécdotas vividas a lo largo de mi carrera científica que podrían hacer reír de lo ridículas que resultan. Todo esto no es un problema legislativo, sino cultural, mucho más lento de resolver y al que sólo hay que darle su peso específico. No creo que ésta sea la verdadera barrera para que la mujer se integre y participe en el sistema de CyT de una forma seria y activa y tenga un peso específico en nuestra Institución. Sólo a través de un trabajo serio y continuado y de una decisión inquebrantable de hacer algo, es como podemos poner de manifiesto nuestra presencia y contribuir al desarrollo y mejora de nuestro sistema de CyT y de nuestra Institución».

Otra científica señala que si bien la discriminación directa, explícita, ha desaparecido, sigue existiendo una discriminación por sexo, y apunta la siguiente experiencia personal: «En el momento en el que me incorporé a mi Centro encontré un ambiente de total igualdad entre los becarios que como yo estaban realizando su Tesis Doctoral, y también entre los investigadores más jóvenes que formaban ya parte de la plantilla del CSIC. Sin embargo, sí encontré una cierta discriminación entre las personas de mayor edad, en general Profesores de Investigación y curiosamente entre el personal de apoyo a la Investigación. En más de una ocasión tuve que explicar que el hecho de ser mujer, casada, con hijos y, en teoría, "sin problemas económicos", no implicaba que mi rendimiento fuera menor que el de cualquier persona que "en teoría" podía dedicar las veinticuatro horas a su labor científica, como a lo largo de los años he demostrado. También recuerdo el día que tuve que prometer al jefe de mi departamento que no tendría más hijos hasta que no terminara la Tesis Doctoral, para que me firmara la petición de una beca predoctoral».

En la actualidad, —prosigue esta misma científica - «la discriminación directa ha desaparecido, pero sigue existiendo una discriminación por sexo, de la que nadie se siente responsable. Pero el resultado de las estadísticas demuestra claramente que la cantidad de hombres en los cargos de designación directa dentro del CSIC es muy superior a la de mujeres (www.csic.es). Sin embargo, la cantidad de mujeres en las primeras etapas de la carrera investigadora es superior al de los hombres y esa proporción va disminuyendo según se avanza en la escala científica. Son mayoría durante el período predoctoral, y postdoctoral y esta proporción disminuye en la primera escala científica (Científico Titular), para ir siendo cada vez menor en las escalas superiores, hasta tal punto que, actualmente, en la escala superior (Profesor de Investigación) en el 


\section{El Área de Ciencia y Tecnología Químicas del CSIC}

área de Química y Tecnología Química del CSIC hay sólo un 10 por 100 de mujeres. No obstante, debo decir que en el momento actual la proporción de mujeres en las escalas superiores es mayor ahora que hace diez años y mucho mayor que hace veinte o treinta años».

Otra investigadora analiza el tema en función de dos vertientes claramente diferenciadas: «La primera de ellas se refiere a los hechos objetivos relacionados con el acceso a la carrera investigadora y el mantenimiento de su actividad profesional. La segunda se refiere al trabajo de investigación desarrollado a través de los años que definen el perfil investigador de un científico En el caso de las mujeres, la vertiente que ofrece mayores diferencias por sexo es la primera. Para hablar de forma detallada de este aspecto, primero es necesario encuadrar a la persona dentro del contexto histórico que marca su generación, ya que los cambios sociales que se han vivido en estos años influyen de forma importante y diferencial en la experiencia personal que haya tenido en el mundo profesional. En mi caso, mi vida profesional comienza en el año 1977, cuando acabo la carrera de Químicas. En ese momento, con la llegada de la democracia hay un replanteamiento de los derechos de las mujeres, que influye de forma importante en el acceso al mundo laboral y en la distribución de los papeles familiares. El modelo familiar mayoritario hasta ese momento, en donde la madre está dedicada al cuidado de los hijos y de la casa y el hombre está dedicado al mundo profesional, cambia para pasar a un modelo en donde las mujeres acceden al mundo laboral y además siguen sintiéndose responsables del cuidado de los hijos y la casa. La incorporación de las mujeres al mundo profesional implicó un cambio importante, tanto a nivel familiar (reparto con la pareja de las tareas domésticas, cambio en el tipo de ingresos, etc.) como social (apertura de guarderías, etc.), abriendo un camino a la igualdad entre los sexos.

En el mundo científico se mantienen en algunos casos valoraciones dependientes del sexo: una mujer siempre tiene que demostrar que es más capaz que cualquier compañero masculino y aunque una mujer soltera es más o menos aceptada, las mujeres casadas o con hijos tienen muchas más dificultades de aceptación.

Dentro del Consejo estos años se caracterizaron por las pocas oportunidades de acceso al Organismo, tanto para hombres como para mujeres. Aunque de forma puntual, en algunos sitios el hecho de ser mujer supuso un hándicap importante en el acceso a la escala científica, una cierta igualdad de oportunidades se mantuvo en muchos Institutos. Esto no ocurrió en otros ámbitos como el de la empresa, en donde el hecho de ser mujer era, y sigue siendo, un hecho discriminatorio». 
Un aspecto importante que señalan varias de las científicas consultadas, y que ya ha sido constatado en otras ocasiones, es que donde sí se mantiene la desigualdad y discriminación es en los cargos directivos y en los de designación directa. Así, tal como señalan: «nuestra Area ha cambiado de forma clara. De una composición en las plantillas en los años 70, en donde las mujeres eran una minoría y no ocupaban ningún cargo de responsabilidad, se ha pasado a una situación en donde la relación numérica entre hombres y mujeres está mucho más equilibrada, manteniéndose la desigualdad en los cargos directivos.

A pesar de que se pueden poner ejemplos en los que la mujer desempeña algún cargo, como el hecho de que en el área haya dos Directoras de Instituto, prácticamente todos los cargos por designación directa, en el Area de Químicas del CSIC son hombres. Como también son hombres la mayoría de los componentes de los tribunales, independientemente de la escala que se juzgue. $\mathrm{Si}$, como se ha dicho anteriormente, hay más mujeres en la etapa de formación, y no se encuentran diferencias entre géneros en cuanto a su aptitud y disponibilidad para la investigación, ¿por qué las escalas científicas superiores y los cargos del CSIC están ocupados principalmente por hombres? ¿Existe realmente discriminación en el CSIC por ser mujer?».

En relación a si existen o no cualidades diferentes entre las mujeres y los hombres para llevar a cabo las tareas científicas, recogemos a continuación algunas de las opiniones recibidas: «Si hablamos del desarrollo del trabajo de investigación, cualidades tales como la imaginación o la habilidad manual (dado que es un área experimental) no tienen género y se encuentran hombres y mujeres que las poseen. Aunque los hombres tengan una mayor tendencia al bricolaje. Sin embargo, quisiera destacar que las mujeres, por lo general, son mucho más voluntariosas, metódicas y constantes que los hombres, por lo que sus trabajos suelen ser más rigurosos y completos. Esto sin menospreciar al género masculino».

«Dentro de la vertiente puramente científica, -opina otra investigadora- creo que las aproximaciones teóóricas y metodologicass que se toman frente al hecho científico no son valorables en función del sexo. No nos preguntamos a qué sexo pertenece el autor de un trabajo científico. Aunque en los últimos tiempos hay quien defiende que los hombres y mujeres tienen un distinto tipo de inteligencia (matemática o emocional), creo que un análisis discriminante de los resultados científicos en función de estas variables no arrojaría resultados significativos».

Otra científica nos comenta: «En mi experiencia personal debo reconocer que, en un principio, yo también sufrí las consecuencias de un cierto rechazo a mi incorporación en la carrera investigadora por mi condi- 


\section{El Área de Ciencia y Tecnología Químicas del CSIC}

ción femenina. Pero quizás fue gracias a esa condición que aprendí a desarrollar mis capacidades de superación para conseguir lo mismo que puede conseguir un hombre, sin renunciar a ninguno de los aspectos que definían mi personalidad. Creo que el esfuerzo que hemos hecho muchas mujeres en ese sentido ha valido la pena.

Hoy ya nadie pone en duda las facultades de la mujer para emprender, desarrollar o dirigir cualquier empresa. Creo que, muy al contrario, hoy somos todos conscientes (y cuando digo "todos» me refiero a hombres y mujeres) de que la mujer tiene incluso a su favor ciertas características que le aproximan, le comunican y le relacionan más directa y profundamente con su entorno. La mujer intuye, observa y siente, con mayor riqueza que el hombre, los aspectos humanos que condicionan el comportamiento de quienes la rodean.

La mujer científica, al igual que el hombre, aplica la lógica como práctica habitual en todos sus actos, decisiones y proyectos. Su visión, de amplio espectro, le permite llegar muy lejos en sus expectativas, y es capaz de medir el alcance de cada una de ellas con los mismos criterios que lo haría un hombre. Pero, a diferencia de él, su espíritu de competitividad no tiene como objetivo el superar al hombre en ninguna de su capacidades, sino más bien en desarrollar su mente para poder aportar una visión convergente desde ángulos diferentes.

No creo que existan diferencias intelectuales en razón del sexo. El género clasifica a las personas, pero sus mentes no responden a clasificación alguna. Cada persona, sea hombre o mujer, observa una conducta acorde con determinados factores o circunstancias, que nada tiene que ver con su género».

Una investigadora del Area de un Instituto de carácter más tecnológico hace el siguiente comentario: «Aunque soy de las que pienso que los hombres y las mujeres son diferentes (qué aburrimiento si así no fuera), no creo que el enfoque de un trabajo, su desarrollo o las conclusiones tengan un enfoque diferente. Sí que he observado, no obstante, que en determinados campos como el de la microscopía electrónica que yo practico, hay tradicionalmente más mujeres que hombres. Mi análisis de la situación es el siguiente:

- Hace falta tener paciencia, virtud tradicionalmente asociada al género femenino.

- El rendimiento publicaciones/hora de trabajo es bajo.

En este tema de las publicaciones, también creo que las mujeres han sido tradicionalmente menos competitivas que los hombres. Además de publicar, las mujeres necesitan estar a gusto en el trabajo. Es algo así como el sexo, que generalmente lo preferimos asociado a la palabra amor. 
También por lo que puedo observar de las relaciones que tiene el Instituto con empresas de todo el mundo, no se puede decir que nuestra Institución y nuestra sociedad sean especialmente machistas. De los científicos o responsables de empresas que vienen a establecer colaboraciones, discutir planes de trabajo o resultados, es rarísimo que venga una mujer. $Y$ las pocas veces que viene alguna mujer nunca vienen solas. Jamás he visto un contrato con una empresa firmado por una mujer. $O$ sea, que para bien o para mal «en todas partes cuecen habas».

Finalmente recogemos parte de una extensa contribución que nos envió una investigadora del Area en la que plantea que ha llegado el momento de hacer un debate nacional sobre nuestro sistema de I+D y que el CSIC debería liderar ese debate.

«Nunca he pensado en Ciencia y Tecnología (CyT) en términos de género. Considero que pensar sobre o hacer buena Ciencia no es cuestión de géneros, sino de preparación y capacidad, y creo que para ello el género femenino como el masculino están igualmente dotados. $\mathrm{Al}$ menos mi experiencia personal en este mundo no me dice lo contrario. Otra cosa son los condicionamientos sociales que influyen de forma diferente en la mujer y en el hombre. Las reglas sociales ponen más vetos a la mujer que al hombre para desarrollar este tipo de actividad. Pero esto no es muy distinto a lo que ocurre en cualquier otra actividad donde el intelecto o, incluso, el prestigio social estén implicados. Tradicionalmente a las mujeres se les asigna menos capacidad de razonamiento, pero creo que esta postura es demasiado tradicional y hoy en día está bastante desprestigiada. Es en este sentido en el que quiero expresar un par de ideas sobre las que creo debemos reflexionar la comunidad científica española, sea del género que sea. Lo anteriormente dicho, a forma de prólogo, creo que es anecdótico y no debe pesar mucho en el ánimo y trabajo de una mujer que le preocupa la situación actual de la Ciencia en este país y concretamente en el CSIC.

Como investigadora del CSIC, un organismo fundamental para la buena marcha de la CyT en este país, creo que ha llegado el momento de plantear un debate nacional sobre nuestro sistema de CyT, y que es el CSIC quien debería liderar este debate. Los años ochenta con la implantación de la Ley de la Ciencia fueron decisivos para que la CyT en nuestro país comenzara a homologarse con el resto de los países europeos. La Ley de la Ciencia creó dos instrumentos esenciales para establecer un sistema moderno de CyT: el Plan Nacional y la Comisión Interministerial de Ciencia y Tecnología (CICYT). No hay que olvidar la Agencia Nacional de Evaluación, una herramienta importante para favorecer la competitividad y transparencia en la financiación de los proyectos del 


\section{El Área de Ciencia y Tecnología Químicas del CSIC}

Plan Nacional. La implantación de la Ley de la Ciencia, junto con el gran esfuerzo realizado por las correspondientes administraciones y grupos de investigación, homologaron la Ciencia española a la media de la Unión Europea. A partir de los años 90, el apoyo al Plan de I+D no se incrementó al ritmo deseado para un correcto desarrollo y crecimiento, e incluso ha sufrido una clara recesión, aunque la inversión se haya mantenido alrededor del 0,87\% del PIB. En mi opinión, después de veinte años de la Ley de la Ciencia nuestro sistema de CyT ha envejecido y está haciendo claramente aguas. Es necesario un nuevo debate y nuevas iniciativas sobre su continuidad, y esto es, en parte, responsabilidad de los profesionales implicados en este sistema, incluidas de forma especial las mujeres.

El CSIC (y me refiero especialmente a sus investigadores/as) como organismo independiente y bien estructurado tiene muchas posibilidades de liderar un debate sobre esta problemática y de iniciar acciones que conduzcan a una forma más inteligente de utilizar los recursos económicos y humanos.

Para terminar con mi visión de género sobre el CyT en el CSIC voy a enumerar algunos ejemplos, que están en boca de todos y cuya solución quizá no requiera ninguna revolución en nuestro sistema de $\mathrm{CyT}$, sino poner un poco de sentido común en su abordaje. A estas consideraciones sí que se las podría atribuir una visión de género. A las mujeres tradicionalmente se les atribuye un sentido mas realista del aprovechamiento de los recursos económicos (por aquello de ama de casa) y, quizás, lo que expongo a continuación pueda considerarse como la visión práctica y limitada de un ama de casa que quiere aprovechar un poco más los recursos de los que dispone para llegar mejor a fin de mes. Por tanto, lo que indico seguidamente no creo que tenga mucho que ver con la alta política científica, sino con introducir un poco de sentido común en temas puntuales de nuestro sistema de CyT y en los que el CSIC puede intervenir sencillamente interesándose por estos problemas. (La gran política se la dejo a nuestros grandes políticos).

Primer tema: La recuperación de nuestros jóvenes cerebros.-Es un tema de continuo debate. El programa de Doctores y Tecnólogos en el extranjero ayudó a formar jóvenes investigadores a un nivel internacional. Después de una gran inversión en este programa se ha descuidado la recogida de los beneficios.

Segundo tema: Los tribunales de selección de jóvenes investigadores.-Siguiendo con la incorporación de estos jóvenes al sistema de CyT y concretamente al CSIC, habría que revisar el sistema de oposiciones. No porque crea que es un mal sistema, sino por el uso que se hace de él. 
La elección de los tribunales que deciden el acceso a la carrera investigadora es esencial. La excelencia o no de la Institución la alcanzan sus investigadores. La selección de los nuevos investigadores es una tarea decisiva para el futuro de la Institución y de la Ciencia española y no creo que se esté haciendo de la mejor forma. Este punto también exigiría una reflexión y debate interno en el CSIC, que echo en falta. Desde luego, su solución o mejora no está en un sorteo.

Tercer tema: Gestión de los fondos de investigación asignados a los investigadores.-¿Es razonable o racional que un grupo de investigadores que lleve cuarenta años demostrando que hace buena Ciencia se quede sin financiación porque otro compañero decide que ahora ya la hace mala? ¿No se debería emplear un poco de tiempo para asegurarse de ello?

En resumen, mi visión de género sobre la CyT en el CSIC, en particular, y en este país, en general, es que le falta un poco de racionalidad y sentido común y que no puede seguir sustentándose sobre las bases de la desconfianza y superficialidad. No es necesario hacer grandes esfuerzos de imaginación, ni tampoco grandes cambios, otros países han ido mejorando su sistema y podrían tomarse como modelos. Estoy segura que otras compañeras /os podrían aportar otros muchos ejemplos sobre los que debatir y que esto no es sólo una responsabilidad de los políticos e instituciones, sino de todos los implicados en el sistema de CyT, incluidas las mujeres. El CSIC tiene una gran responsabilidad en todo esto, pues al contrario que las Universidades, que además de investigar deben cumplir su función docente, nuestra Institución sólo tiene como misión investigar».

\section{Conclusiones}

Conjugando los datos estadísticos mostrados con las aportaciones personales recogidas, se pueden extraer las conclusiones generales que se resumen a continuación:

El Area de la Ciencia y la Tecnología Químicas presenta una situación alentadora sobre la presencia de las mujeres, aunque mejorable. Aunque estamos lejos de ofrecer datos igualitarios por sexo, parece darse una tendencia hacia ello, según lo sugieren los testimonios de las investigadoras a las que hemos consultado. Aunque, en conjunto, no hay diferencias significativas entre Institutos, el caso del Instituto de Química Médica aparece como una excepción, pues las mujeres son mayoría entre el personal científico. Las desigualdades por sexo se acentúan, en general, a medida que se asciende en la escala de reconocimiento académico 


\section{El Área de Ciencia y Tecnología Químicas del CSIC}

y científico. Al mismo tiempo, la presencia de científicas del Area en cargos de libre designación no muestra tendencia alguna a la igualdad.

$\mathrm{Al}$ margen de las conclusiones expuestas, deducidas a partir de este trabajo, cabe hacer las siguientes consideraciones:

Si son excepción los artículos de Ciencia y Tecnología Química que por si mismo constituyen un trabajo completo sobre un determinado tema de investigación, éste que nos ocupa está lejos de contarse entre ellos. Por otra parte, no es sorprendente encontrar que el análisis de los mismos datos experimentales conduzca a diferentes conclusiones, de acuerdo con la especialización, experiencia e, incluso, imaginación del investigador que los analice. Este artículo no es más que una breve panorámica del lugar que ocupan las químicas dentro del Area de Ciencia y Tecnología Químicas del CSIC, vista por un limitado número de científicas que desarrollan su labor investigadora dentro de este hábitat. El artículo se ha ceñido a presentar unos datos generales y objetivos de la realidad actual, junto con opiniones particulares de ocho científicas sobre el tema, salpicadas de sus experiencias vividas a lo largo de su trayectoria. Para algunas, el camino habrá sido, o, es, más o menos, cuesta arriba. Para otras, más llano. No obstante, parece justo reconocer que , tanto quienes han contribuido a la elaboración de éste artículo con sus aportaciones personales, como aquellas cuyo esfuerzo se refleja en una simple cifra estadística, han hecho camino al andar.

Por todas las consideraciones expuestas, este artículo no es un capítulo cerrado y es al lector individual, independientemente de su género, al que le corresponde extraer sus propias conclusiones. 